UCRL-JC-111188

PREPRINT

\title{
3D Simulations of an Electrostatic Quadrupole Injector
}

D. P. Grote, A. Friedman, and S. Yu

This paper was prepared for submittal to the Proceedings of the Computational Accelerator Physics Conference

Pleasanton, California

February 22-26, 1993

\section{February 1993}

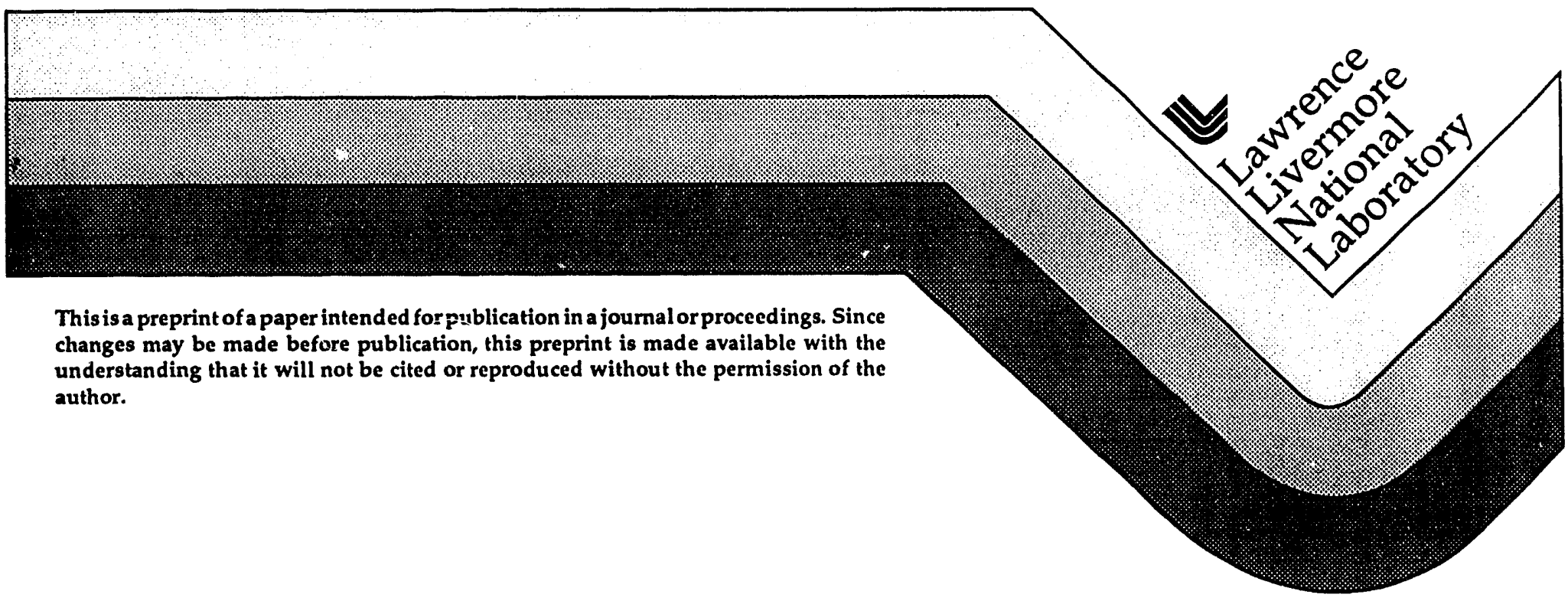




\section{DISCLAIMER}

This document was prepared as an account of work sponsored by an agency of the United States Government. Neither the United States Government nor the University of California nor any of their employees, makes any warranty, express or implied, or assumes any legal liability or responsibility for the accuracy, completeness, or usefulness of any information, apparatus, product, or process disclosed, or represents that its use would not infringe privately owned rights. Reference herein to any specific commercial product, process, or service by trade name, trademark, manufacturer, or other wise, does not necessarily constitute or imply its endorsement, recommendation, or favoring by the United States Government or the University of California. The views and opinions of authors expressed herein do not necessarily state or reflect those of the United States Government or the University of California, and shall not be used for advertising or product endorsement purposes. 


\title{
3D SIMULATIONS OF AN ELECTROSTATIC QUADRUPOLE INJECTOR*
}

\author{
David P. Groie \\ University of California at Davis \\ Lawrence Livermore National Laboratory, Livermore CA 94550 \\ Alex Friedman \\ Lawrence Livermore National Laboratory, Livermore CA 94550 \\ Simon $\mathrm{Yu}$ \\ Lawrence Berkeley Laboratory, University of California \\ Berkeley California 94720
}

\begin{abstract}
Analysis of the dynamics of a space charge dominated beam in a lattice of electrostatic focusing structures requires a full three-dimensional code that includes self-consistent space charge fields and the fields from the complex conductor shapes. The existing WARP3 $\mathrm{d}^{1-3}$ code, a particle simulation code which has been developed for HIF applications, contains machinery for handling particles in three-dimensional fields. A successive overrelaxation field solver with subgrid-scale placement of boundaries for rounded surfaces and four-fold symmetry has been added to the code. The electrostatic quadrupole (ESQ) injector for the ILSE ${ }^{4}$ accelerator facility being planned at Lawrence Berkelay Laboratory is shown as an application. The issue of concern is possible emittance degradation because the focusing voltages are a significant fraction oi the particles' energy and because there are significant nonlinear fields arising from the shapes of the quadrupole structures.
\end{abstract}

\section{INTRODUCTION}

WARP3d is a three-dimensional particle-in-cell code designed for heavyion fusion (HIF) applications. For many of these problems, it is necessary to model the transport of space charge dominated, heavy-ion beams over long distances. For many simulations of this type, the external focusing elements are modeled as having uniform, sharp edged fields.

Simulations of runs over shorter distances, in particular simulations of the ESQ injector, revealed the need for more accurate, self-consistent, focusing elements which include image, fringe, and other non-linear fields. That has been accomplished by placing the conductors that make up the focusing elements within the grid and including them in the field solution. Simple, widely spaced conductors were first implemented; they were dealt with via a capacity matrix method..$^{5,6}$

More general conductors, such as the closely spaced, "inter-digital" quadrupole focusing conductors of the ESQ, are dealt with via a point successive overrelaxation (SOR) method. ${ }^{7}$ This was done because, for the more complex

*This work was performed under the auspices of the U.S. D.O.E. by Lawrence Livermore National Laboratory under contract W-7405-ENG-48. 
structures the capacity matrix becomes too big for solution in a reasonable amount of time. The matrix becomes bigger when the structures take up more space inside the field grid and require more points to be described. Also, the elements cannot be treated individually so all must be included in one very large matrix. Currently, the input to the code is set up to specifically handle the "inter-digital" structure. The internal representation however, can handle any conductor shape.

Use of four-fold symmetry of the potential $\phi$ puts the centerline of the beam along the edge of the grid. The normal derivative is set to zero on the symmetry boundaries. The particles still fill the whole system, but they all contribute their charge to, and get the fields from, the one simulated quadrant.

\section{SOR METHOD AS IMPLEMENTED IN WARP3D}

In three dimensions, Poison's equation is approximated by a seven point finite difference scheme. After rearrangement and inclusion of the relaxation parameter, the equation that is iterated for each grid point is:

$$
\begin{aligned}
\phi_{i j k} \Leftarrow & \omega \Delta^{2}\left(\rho_{i j k} / \epsilon_{0}+\frac{\phi_{i+1, k}+\phi_{i-1, k}}{\Delta x^{2}}+\frac{\phi_{i j+1 k}+\phi_{i j-1 k}}{\Delta y^{2}}+\frac{\phi_{i j k+1}+\phi_{i j k-1}}{\Delta z^{2}}\right) \\
& +(1-\omega) \phi_{i j k} .
\end{aligned}
$$

where the subscripts are grid cell indices, $\omega$ is the relaxation parameter,

$$
\frac{1}{\Delta^{2}}=\frac{1}{\Delta x^{2}}+\frac{1}{\Delta y^{2}}+\frac{1}{\Delta z^{2}}
$$

and $\Delta x, \Delta y$, and $\Delta z$ are the grid cell sizes in the various directions. The ordering that is used is even-odd, a straightforward three-dimensional generalization of the familiar red-black scheme.

In the code, for efficiency, this equation is applied to the entire potential array excepting the first and last axial planes. This is done by treating the $3 \mathrm{~d}$ array as a 1d array and applying the equation to every other point. However, this equation is not correct at the boundaries, thus, before this equation is applied, the potential on the grid boundaries, and the conductor boundaries if needed, is saved. The potential on points internal to the conductors is enforced by setting them to the desired value before each iteration.

\section{SUBGRID-SCALE PLACEMENT OF BOUNDARIES}

Subgrid-scale placement of boundaries is handled by explicitly including the boindary location in the finite difference form of Poison's equation. In 1-D, the finite difference form of Poison's equation, $\nabla^{2} \phi=-\rho / \epsilon_{0}$, is

$$
\frac{\phi_{i+1}-2 \phi_{i}+\phi_{i-1}}{\Delta x^{2}}=-\frac{\rho_{i}}{\epsilon_{0}} \text {. }
$$

If the edge of the conductor surface is between the grid points $i$ and $i+1$, the potential at the edge would be interpolated as

$$
\phi_{\text {edge }}=(1-\delta) \phi_{i}+\delta \phi_{i+1} .
$$

Here, $\delta$ is the distance between the grid point $i$ and the surface, divided by the grid cell size. The value of $\phi_{\text {edge }}$ is the known voltage on the conductor, and the value $\phi_{i+1}$ is a free parameter since it is inside the conductor. The previous equation can be rearranged to give a value to $\phi_{i+1}$.

$$
\phi_{i+1}=\frac{\phi_{\text {edge }}-(1-\delta) \phi_{i}}{\delta}
$$


This is then put back into Poison's equation,

$$
\frac{\left(\phi_{\text {edge }}-(1-\delta) \phi_{i}\right) / \delta-2 \phi_{i}+\phi_{i-1}}{\Delta x^{2}}=-\frac{\rho_{i}}{\epsilon_{0}}
$$

Note that in the limit $\delta \rightarrow 0, \phi_{i}$ approaches $\phi_{\text {edge }}$. This is then rearranged to bring all of the terms in $\phi_{i}$ to the left hand side, and the SOR relaxation parameter, $\omega$, is included to give the final equation:

$$
\phi_{i}=\left(\frac{\phi_{i-1}+\phi_{\text {edge }} / \delta+\Delta x^{2} \rho_{i} / \epsilon_{0}}{2+\frac{1-\delta}{\delta}}\right) \omega+\phi_{i}(1-\omega) .
$$

This equation is used for the point $\phi_{i}$ during iteration. The original equation is still used everywhere else.

The idea extends easily to two and three dimensions. The three-dimensional version has been implemented in WARP3d. The seven point difference scheme is used and any points that are inside the conductor are handled as above. For the allowed classes of conductors, there are eight cases, with up to three, non-opposite, nearby points inside the conductor. The case when one point is in the conductor is handled as above but with the differences in the other two directions included. For example, if the point that is in the conductor is $\phi_{i+1 j k}$, the equation for $\phi_{i j k}$ would look like

$$
\begin{aligned}
\phi_{i j k}= & \frac{\omega \Delta^{2}\left(\rho_{i j k} / \epsilon_{0}+\frac{\phi_{e d g e} / \delta_{x}+\phi_{i-1, k}}{\Delta x^{2}}+\frac{\phi_{i j+1 k}+\phi_{i j-1 k}}{\Delta y^{2}}+\frac{\phi_{i, j k+1}+\phi_{i j k-1}}{\Delta z^{2}}\right)}{1+\frac{\left(1-\delta_{x}\right) \Delta^{2}}{\delta_{x} \Delta x^{2}}} \\
& +(1-\omega) \phi_{i j k} .
\end{aligned}
$$

Here, $\delta_{x}$ is the unitless distance in the $\mathrm{x}$ direction between the grid point $i j k$ and the surface.

When there are two points inside the conductor, the interpolations are done for each of the two points and then put back into the finite difference equation. If the points $\phi_{i+1 j k}$ and $\phi_{i j+1 k}$ are inside the conductor, for example, the equation for $\phi_{i j k}$ would be

$$
\begin{aligned}
\phi_{i j k}= & \frac{\omega \Delta^{2}\left(\rho_{i j k} / \epsilon_{0}+\frac{\phi_{e d g e} / \delta_{x}+\phi_{i-1, k}}{\Delta x^{2}}+\frac{\phi_{e d g e} / \delta_{x}+\phi_{i j-1 k}}{\Delta y^{2}}+\frac{\phi_{i j k+1}+\phi_{i j k-1}}{\Delta z^{2}}\right)}{1+\frac{\left(1-\delta_{x}\right) \Delta^{2}}{\delta_{x} \Delta x^{2}}+\frac{\left(1-\delta_{y}\right) \Delta^{2}}{\delta_{y} \Delta y^{2}}} \\
& +(1-\omega) \phi_{i j k} .
\end{aligned}
$$

Here, $\delta_{x}$ and $\delta_{y}$ are the respective unitless distances in the $\mathrm{x}$ and $\mathrm{y}$ directions between the grid point $i j k$ and the surface. The extension to three points within the conductor is similar.

In the code, to ease bookkeepping, there are two sets of arrays for the points near the conductor surfaces: one set for the points that have $i+j+k$ even, and one for the points that have $i+j+k$ odd. The values that are saved in the arrays are the location of the point, the magnitude of the distances from the surface in the $\mathrm{x}, \mathrm{y}$, and $\mathrm{z}$ directions, the sign of the direction to the point that is inside the conductor, and the previous value of the potential at the point.

In the simulations of the ESQ, without the subgrid-scale placement of boundaries, small grid cell sizes are needed to resolve the surfaces of the conductors. Without resolving the conductors, the focusing fields and therefore the beam envelope were inaccurate. The emittance growth, which is very sensitive to the shape of the envelope, was then also incorrect. Subgrid-scale placement of boundaries alleviated this problem since the focusing fields are 
correct, almost independent of the grid cell size. The restriction on the grid cell size is then greatly relaxed.

\section{INJECTOR DESCRIPTION}

The injector must produce a 2 megavolt, $0.8 \mathrm{Amp}$ beam with low normalized transverse emittance, near $0.5 \pi$-mm-mrad. The source emits into a diode region, or gap, with a potential drop; the beam then enters the first quadrupole, then passes through several more quadrupoles with common end plates, and exits the injector. These quadrupoles have an "inter-digital" structure; two rods placed along the axial direction are attached to the left plate and two to the right plate, with the sets of rods overlapping.

With the high currents needed from the injector, large focusing fields are needed to confine the beam. Since the particles have low energy in the injector, the focusing potentials are a large fraction of the particle energy. This results in what is called the "energy effect". It is significant here because, at low energy, the motion is not paraxial. Note that this affect is present in all electrostatic quadrupoles, but the focusing fields are generally much smaller then the particle energy so the resulting distortion is very small.

The focusing structure produces fields that are not purely quadrupolar. A variety of multipoles arise of the form $\phi_{n m}=V_{n m}(r / R)^{n} \cos (m \theta)$, where $\mathrm{R}$ is the quadrupole aperture and $\phi=\sum_{n, m} \phi_{n m}$. The quadrupole field is the $\phi_{22}$ term and the octupole term is $\phi_{44}$.

Several injector designs have been studied. Because of breakdown, the voltages that can be applied to the conductors are limited. The maximum voltage that can reasonably be applied across the diode is 1 Megavolt, but $0.5 \mathrm{MV}$ would be better. Parameters are given in table 1 , along with those of a scaled experiment that is being carried out at LBL, which also has been simulated.

\section{DESCRIPTION OF COMPUTER RUNS}

The beam is injected into the diode with constant current from a planer injection surface which is assumed to be an equipotential and is followed to the end of the injector. The runs are quasi time dependent; for efficiency, the time interval between field solutions is longer than the time step by which the particles are advanced. The transient behavior of the particles is lost and only steady-state is obtained. Generally, steady-state is reached shortly after one transit time across the injector.

The size of the field grid cells is limited by the accuracy of the selffields. With the subgrid-scale placement of the bounciaries, the fields from the conductors are accurate with fairly large grid cell sizes. The field grid must be large enough to include enough of the quadrupole conductors for good representation of the focusing fields. To meet these requirements, on the order of 50 grid cells are needed in each tranverse direction; the beam occupies about a third and at least half of the rod is inside the grid. The number of grid cells in the axial direction, between two hundred and six hundred, depends on the number of quadrupoles being simulated.

The injected particle distribution is a semi-Gaussian with circular transverse profile. Typically, the total number of particles used ranges from 70,000 to 300,000 , with several hundred injected at each time step. The transit time of typical runs is between 300 and 1000 time steps, depending on the time step 


\begin{tabular}{|c|c|c|}
\hline & ILSE injector & Scaled experiment \\
\hline Beam current (Amps) & 0.8 & 0.008 \\
\hline Inital beam radius $(\mathrm{cm})$ & 6.0 & 10.0 \\
\hline \multirow[t]{2}{*}{ Quad voltages $^{a}(\mathrm{kV})$} & $220,260,260$ & $10.3,12.2,12.2$ \\
\hline & $255,255,250$ & 11.97 \\
\hline Quad length ${ }^{b}$ of first quad $(\mathrm{cm})$ & 31.0 & 7.15 \\
\hline of others $(\mathrm{cm})$ & 47.0 & 11.155 \\
\hline Rod length of first quad $(\mathrm{cm})$ & 25.5 & $5.89,6.195^{c}$ \\
\hline of others $(\mathrm{cm})$ & 39.5 & 9.7 \\
\hline Apertures $^{d}(\mathrm{~cm})$ & $13,12,11$ & $3.25,3.0,2.75$ \\
\hline & $10,9,8$ & 2.5 \\
\hline Plate width $(\mathrm{cm})$ & 1. & 0.6 \\
\hline
\end{tabular}

${ }^{a}$ The ILSE injector has either four, five, or six quads depending on the diode voltage. The scaled injector has four quads.

${ }^{b}$ Distance between center of plates.

'The rods of the first quad are not the same length.

${ }^{d}$ Distance from beam center to the rod. Rod radii are $8 / 7$ of the aperture.

Table 1: Parameters for ILSE injector and scaled injector experiment.

size. Another 100 to 200 steps are done to ensure convergence. The number of cycles, or field solves, is five to ten. The shortest runs with fewer time steps and particles used just under 3 minutes of CPU time on the NERSC Cray C-90 machine. The longer runs done for checks of numerical errors use 20 to 30 minutes.

\section{ILSE INJECTOR}

The first simulations that were done were of the ILSE injector with a 0.5 MV diode. The initial run showed a very large emittance growth. Figure 1 shows the normalized emittance along with the beam profiles and potential drop on axis. Note that the sharp rise in emittance in the $y$ direction follows shortly after the widest part of the $y$ envelope, and similarly in the $x$ direction.

This emittance growth is not true emittance growth, but rather results from the twisting or "essing" of phase space, which can be undone. The $y-y^{\prime}$ phase space at several points along $z$ are shown in figure 2. The shape of the phase space, the essing, indicates action of fourth order (in potential) fields, $\phi_{4 m}$. The same runs as above were done, but cancelling out the various fourth order fields by applying their negative (calculated via a least squares fit). Cancelling the pure octupole field, $\phi_{44}$, and the $\phi_{40}$ field had little effect on the emittance growth. Cancelling the $\phi_{42}$ field however, had a dramatic effect on the emittance, which was cut in half.

Both $V_{40}$ and $V_{44}$ change sign over one quadrupole element. Coupled with the fact that the energy changes little inside a quadrupole, their effect on the beam nearly vanishes with integration over one quadrupole. $V_{42}$, on the other hand, does not change sign over one quadrupole element. With integration over one quadrupole, its effect builds up. However, its sign does change from quadrupole to quadrupole, partially cancelling itself, leading to the reduction in emittance. The cancellation is not complete since the envelope and the 

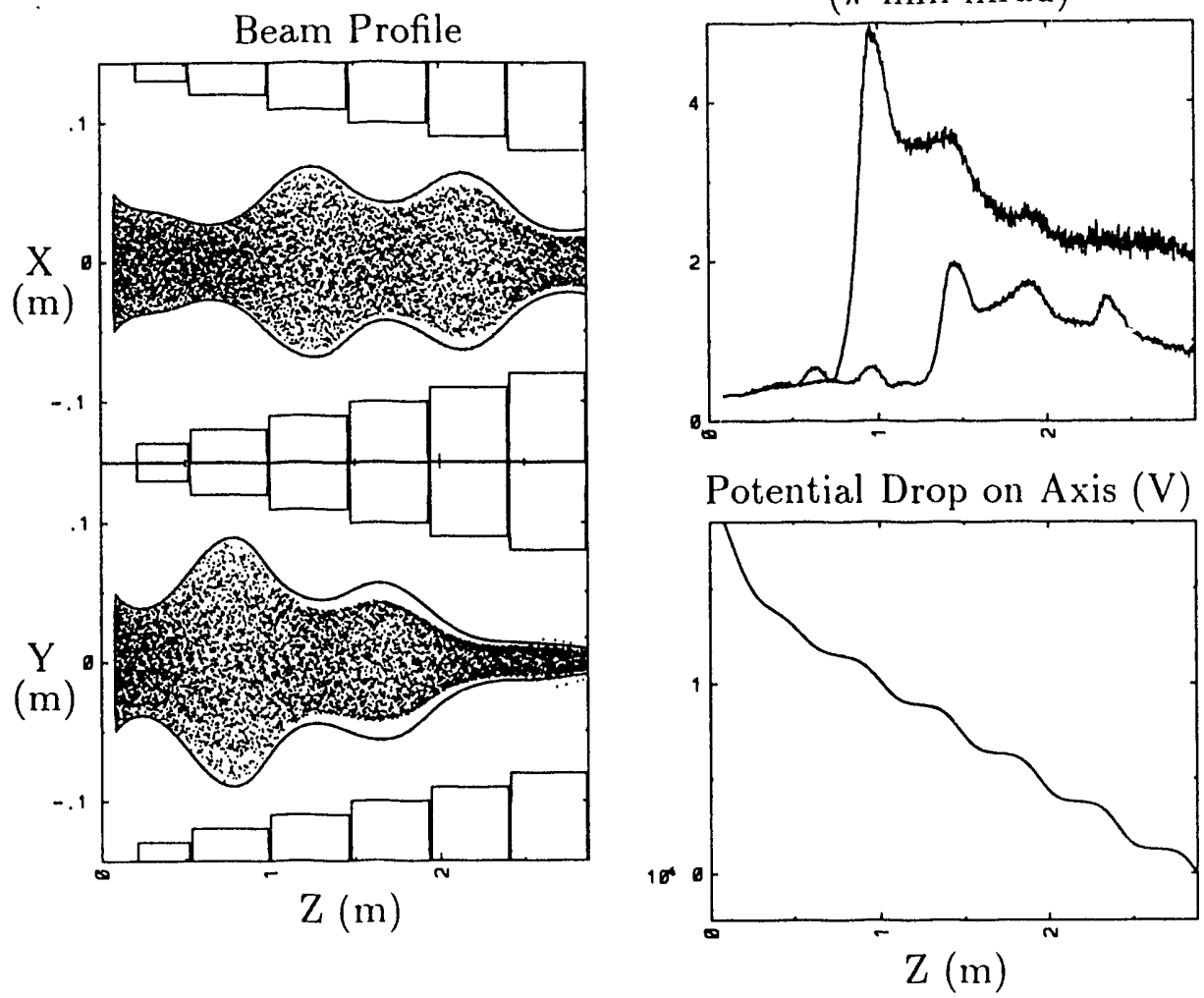

Figure 1: ILSE injector results. The top curve in the emittance corresponds to the $y$ profile. Note that the peak in emittance follows shortly after the profile has reached its widest. The curves surrounding the beam are the RMS envelopes. The gap between the envelope and the particles is due to beam hollowing. The rectangles are the quadrupoles.

energy of the beam change.

The remaining emittance growth is caused by the energy effect. That effect was analyzed by expanding to fourth order in potential the equation of motion of a particle in the fields. The resulting expression for the perturbation in $v_{x}$ is

$$
\begin{aligned}
& \frac{d v_{x 1}}{d z}=\frac{v_{z 0}}{2}\left\{\left[\frac{1}{8}\left(\frac{V_{22}}{T_{0} / e}\right)^{2}+\left(\frac{V_{44}}{T_{0} / e}\right)\right]\left[-\frac{\partial}{\partial x}\left(\frac{r}{R}\right)^{4} \cos 4 \theta\right]+\right. \\
& {\left[\frac{1}{2}\left(\frac{V_{22}}{T_{0} / e}\right)\left(\frac{V_{20}}{T_{0} / e}\right)+\left(\frac{V_{42}}{T_{0} / e}\right)\right]\left[-\frac{\partial}{\partial x}\left(\frac{r}{R}\right)^{4} \cos 2 \theta\right]+} \\
& {\left.\left[\frac{1}{8}\left(\frac{V_{22}}{T_{0} / e}\right)^{2}+\frac{1}{4}\left(\frac{V_{20}}{T_{0} / e}\right)^{2}+\left(\frac{V_{40}}{T_{0} / e}\right)\right]\left[-\frac{\partial}{\partial x}\left(\frac{r}{R}\right)^{4}\right]\right\} }
\end{aligned}
$$

The terms in $V_{2 m}$ arise from the interaction of the terms $\frac{\partial \phi}{\partial x}$ and $\frac{1}{v_{z}}$ which represents the energy effect. The terms in $V_{4 m}$ arise from $\frac{\partial \phi}{\partial x}$, the quadrupole structure.

The energy effect can be cancelled like the fourth order fields by applying 

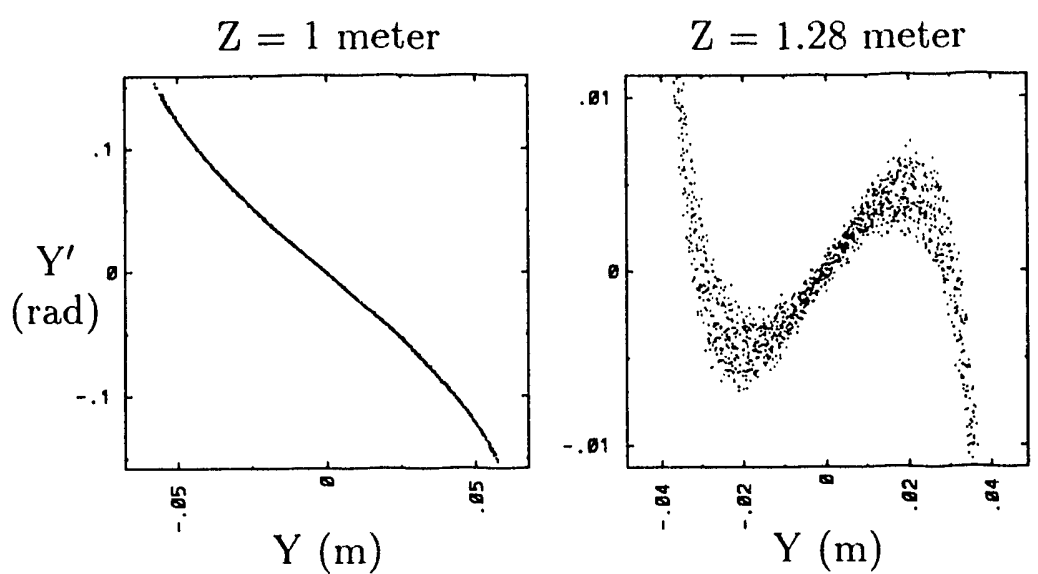

Figure 2: ILSE injector phase space at various locations along the injector.

Normalized RMS Emittance

$(\pi-\mathrm{mm}-\mathrm{mrad})$

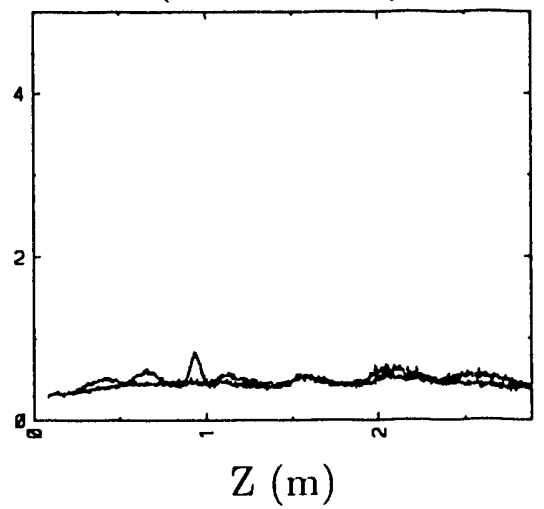

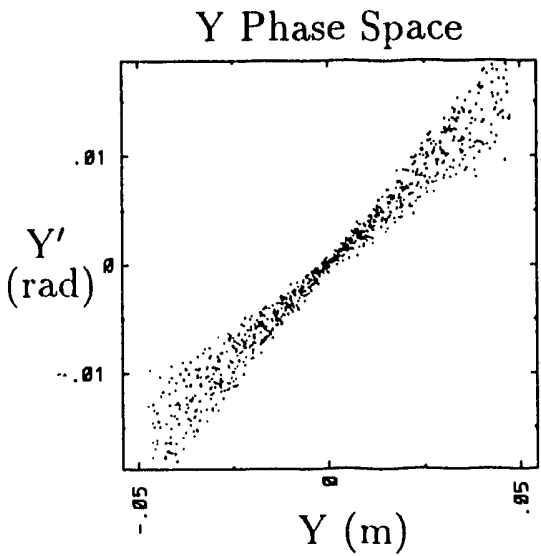

Figure 3: Injector results with both corrections. The emittance becomes flat and the essing of the phase space goes away. The "bow tie" shape in the phase space results from using particles from a finite range of $z$.

the appropiate fields to the beam. The correcting fields should, on integration over one quadrupole, cancel the energy effect. Over one quadrupole, the term $V_{22} V_{20}$ changes sign; its integral nearly vanishes. The term $\left(V_{20}\right)^{2}$ is small compared to $\left(V_{22}\right)^{2}$ and so can be ignored. This leaves only $\left(V_{22}\right)^{2}$ as significant. The correcting fields that are then needed are $V_{44}^{\text {cor }}$ and $V_{40}^{\text {cor }}$. The correcting fields reduce the emittance growth also by nearly a factor of two.

One more run was done where both corrections were done; the fourth order fields were cancelled and the energy effect was corrected. The plot of the emittance, figure 3 , shows that there is no emittance growth and all of the essing in the phase space has disappeared.

The injector was also simulated with two other diode energies, $1 \mathrm{MV}$ and $0.75 \mathrm{MV}$. As expected, the growth in both cases is much less than with the $0.5 \mathrm{MV}$ diode. Application of the correcting fields reduced the emittance as in the $0.5 \mathrm{MV}$ case.

\section{SELF-CONSISTENT CORRECTIONS}

The goal of these simulations is to reduce the emittance from the injector 
to an acceptable level. We have shown the cause of the growth in the above section and have demonstrated that it can fairly easily be removed. We now wish to remove the emittance by self-consistent means. This is done by changing the quadrupole's structure. Two changes that were made to the quadrupoles where to move all of the rods in or out to change the aperture, and to move two rods in and two out, maintaining the same average aperture. The voltages on the quadrupoles were also varied.

Moving all of the rods in or out and changing the voltages changed the focusing fields and therefore the envelope. Many runs were done, varying the apertures to reduce the maximum size of the envelope. The optimum apertures for the injector with the $1 \mathrm{MV}$ diode were found. The emittance was reduced to nearly the source emittance, so no other adjustments are needed.

The injector with the $0.5 \mathrm{MV}$ diode showed considerable emittance growth. By moving all of the rods in or out and changing the voltages, the emitance was reduced somewhat, though a growth of nearly a factor of two remained. To reduce the emittance further, the rods needed to be moved in and out, affecting only the fourth order fields. A sizeable number of runs have been made, doing both parameter scans and ad-hoc searches for minimal emittance. The emittance has to date been reduced down to one and a half times the inherent source emittance.

\section{CONCLUSIONS}

The methods described herein have allowed use of much larger grid cell sizes while still maintaining accurate fields from the conductors. This has made possible parameter scans and searches for minimal emittance.

The simulations of the ESQ injector have shown that much of the emittance growth is caused by fourth order fields. The speed of the code has allowed many runs to be done to study the system and to minimize the emittance. We find these three-dimensional simulations to be an effective design tool.

\section{REFERENCES}

${ }^{1}$ Alex Friedman, et. al., these proceedings

${ }^{2}$ Debra Callahan, et. al., these proceedings

${ }^{3}$ A. Friedman, D. P. Grote, and I. Haber, "Three-dimensional particle simulation of heavy-ion fusion beams," Phys. Fluids B 4, 2203 (199:).

${ }^{4}$ T. Fessenden, R. Bangerter, D. Berners, J. Chew, S. Eylon, A. Falte'.ls, W. Fawley, C. Fong, M. Fong, K. Hahn, E. Henestroza, D. Judd, E. Lee, C. Lionberger, S. Mukherjee, C. Peters, C. Pike, G. Raymond, L. Reginato, H. Rutkowski, P. Seidl, L. Smith, D. Vanecek, S. Yu, F. Deadrick, A. Friedman, L. Griffith, D. Hewett, M. N Newton, and H. Shay, "ILSE, The Next Step toward a Heavy Ion Induction Accelerator for Inertial Fusion Energy," Proc. 14th Int. Conf. on Plasma Physics and Controlled Nuclear Fusion Research, IAEA, Wurzburg, Germany, Sept. 30-Oct. 7, 1992.

${ }^{5}$ Charles K. Birdsall and A. Bruce Langdon, Plasma Physics via Computer Simulation, McGraw-Hill Book Company, 1985

${ }^{6}$ D. P. Grote, A. Friedman, and I Haber, "3d Simulations of Axially Confined Heavy Ion Beams in Round and Square Pipes" Particle Accelerators, 3738, 141, (1992)

${ }^{7}$ William H. Press et. al., Numerical Recipes: The Art of Scientific Computing Cambridge University Press, 1986 

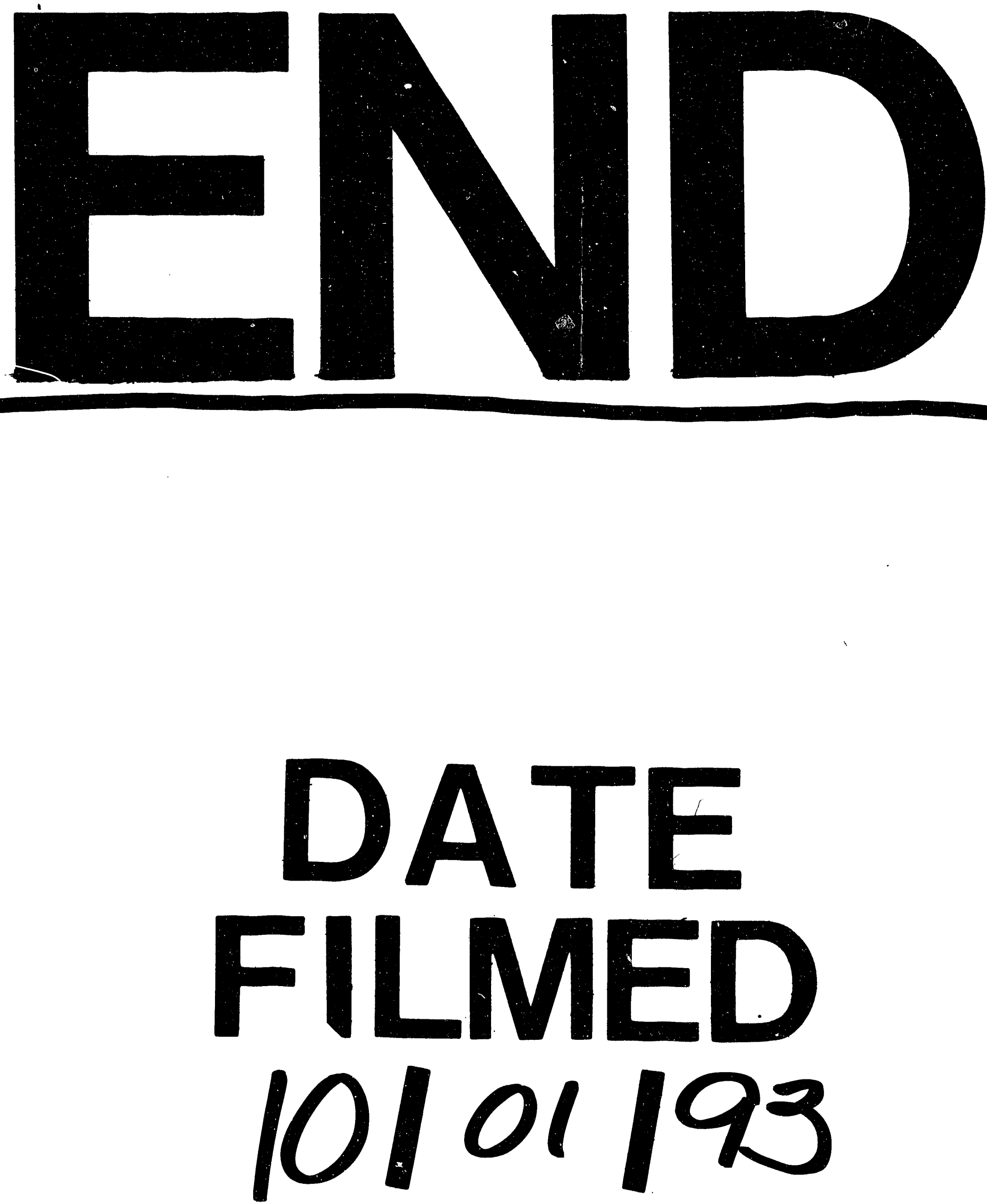
